\title{
Focus on Nematodes: Microscopic Roundworms
}

Gary Bauchan $^{1}$, Joseph Mowery ${ }^{1}$, Ron Ochoa ${ }^{1}$, Eric Palevsky ${ }^{2}$ and Lynn Carta ${ }^{1}$

${ }^{1}$ US Department of Agriculture, Agricultural Research Service, Beltsville, Maryland, United States, ${ }^{2}$ Israel Agricultural Research Organization, Ramat Yishay, Tel Aviv, Israel

Phytoparasitic nematodes or plant-parasitic nematodes are tiny, slender roundworms; typically, between 15-35 microns wide and 250 microns to $12 \mathrm{~mm}$ in length [1]. Most of the agriculturally important nematodes are parasites of plants which cause over $\$ 100$ billion in global crop losses [2]. The body is often ornamented with various ridges (annulations), lateral lines, bristle or other distinctive structures. Nematodes are often described as a tube within a tube due to the digestive system following from the mouth to the anus. Nematodes are typically extracted from soil, roots or other plant material using a Baermann funnel or grown in cultures and then mounted in a medium on a microscope slide with a coverslip. Observing nematodes using polarization or Differential Interference Contrast DIC microscopy improves contrast for imaging external structures such as oral lips and phasmids (secretory organs), number of annulations, shape and position of the vulva (female genital region) and spicules (male genital structures), location of anus, and shape of the tail.. Since nematodes are translucent, internal structures can also be identified including the length of the feeding stylet. the shape of the knobs at the base of the stylet the position and shape of the digestive and secretory organs, the reproductive organs of males and females as well as a set of measurement ratios called the DeMan Indices [3] . SEM imaging of nematodes started with the classical system of fixation, dehydration, critical point drying and sputter coating; however, due to the soft-bodied nature of the worms many shriveled and did not retain important diagnostic features. Low-temperature SEM (LT-SEM) images preserve the turgidity and the critical morphological structures required for identification of nematodes. We have developed several techniques for preparing nematodes for observation in the LT-SEM including picking up live nematodes with an eyelash and placing them on carbon sticky tabs, pipetting liquid cultures onto filter paper and then allowing them to dry briefly prior to contact freezing in liquid nitrogen [4]. A high angle azimuth plant holder has been used to obtain lateral views of nematodes. Recently a feeding arena was designed to record videos of predatory mites feeding on nematodes prior to flash freezing, capturing the native feeding behavior for observation in the LT-SEM. Initial studies have also begun to observe internal structures of nematodes using a confocal laser scanning microscope. These modern microscopy techniques will be discussed as a multi-faceted system to observe these agriculturally important nematodes and linking behavior with their biology.

References

[1] K. Lambert and S. Bekal. The Plant Health Instructor. (2002) doi:10m1094/PHI-I-202-1218-01.

[2] J.P. McCarter. Plant Cell Monographs 15 (2009) p. 239-267 dio:10.1007/7089

[3] G. Thorne, Proc. Helmithological Soc. Washington 16 (1949) p.37-73

[4] L.K. Carta, et al. J Nematology 42 (2010) p.46-54 